\title{
Nucleolar dominance: a model for rRNA gene silencing
}

\author{
Brian McStay ${ }^{1}$ \\ Biomedical Research Centre, University of Dundee, Ninewells Hospital, Dundee DD1 9SY, United Kingdom
}

Research over the last decade has demonstrated that the regulated packaging of DNA into chromatin is fundamental to keeping genes in an active/open or a more condensed/inactive conformation. Nucleosomes, the basic repeating unit of chromatin, contain two molecules each of canonical histones, H2A, H2B, H3, and $\mathrm{H} 4$. These are organized as an $\mathrm{H} 3-\mathrm{H} 4$ tetramer associated with two $\mathrm{H} 2 \mathrm{~A}-\mathrm{H} 2 \mathrm{~B}$ dimers. The $\mathrm{NH}_{2}$ termini of histones protrude from the nucleosome, and are the target for a wide array of covalent modifications including acetylation, methylation, and phosphorylation. These modifications are applied and removed in a highly specific manner to generate what has been described as the histone code (Jenuwein and Allis 2001). This code is, in turn, read by chromatin-associated factors. For example, individual acetylated lysine residues are recognized by factors with bromodomains and methylated lysines by chromodomain-containing proteins. Additionally, the action of ATP-dependent chromatin remodeling activities can displace nucleosomes altering the accessibility of DNA within chromatin templates (Flaus and OwenHughes 2001). While most of this work has focused on protein-encoding genes transcribed by RNA polymerase II, more recent studies, including one by Earley et al. (2006) in this issue of Genes \& Development, are aimed at elucidating the role that chromatin plays in determining the activity status of the genes that encode ribosomal RNAs (rRNAs).

Eukaryotic genomes contain many rRNA gene copies, ranging from hundreds to thousands in some plants, organized in tandem arrays. rRNA genes are transcribed by RNA polymerase I (Pol I) into a precursor RNA (prerRNA) that encodes the three largest RNA components of ribosomes. Pre-rRNA coding sequences are separated by intergenic spacers (IGS) that can range in size from 3 $\mathrm{kb}$ in yeast to $30 \mathrm{~kb}$ in mammals. Pre-rRNA synthesis is regulated by elements including promoters, transcriptional enhancers, and terminators that are located within the IGS (Grummt 1999). Transcription of rRNA

\footnotetext{
${ }^{1}$ Correspondence.
}

E-MAIL brian.mcstay@cancer.org.uk; FAX 44-1382-669993.

Article and publication are at http://www.genesdev.org/cgi/doi/10.1101/ gad.1436906. gene arrays results in formation of a nucleolus; consequently, they are termed nucleolar organizer regions (NORs). Importantly, not all NORs are transcriptionally active. Species with multiple NORs can vary the active proportion.

Pre-rRNA is matured into $18 \mathrm{~S}, 5.8 \mathrm{~S}$, and $28 \mathrm{~S}$ rRNA by a precisely ordered series of events that includes both cleavages and targeted base modifications. These are required to ensure correct folding of rRNAs for ribosome assembly and function. These multiple steps in ribosome biogenesis require a bewildering array of proteins and small nucleolar RNAs that converge on transcriptionally active NORs, forming such a high concentration of ribonucleoprotein complexes that nucleoli are the most prominent feature of the eukaryotic nucleus.

The electron micrographs of Miller and Beatty (1969) demonstrated that the coding regions of active repeats are fully loaded with Pol I. rRNA gene transcription accounts for $\sim 50 \%$ of nascent RNA synthesis in a cell. This high density of transcription and the recent demonstration that it is tightly coupled to pre-rRNA processing (Granneman and Baserga 2004) argues that rRNA gene chromatin needs to be especially accessible. As cells approach metaphase, Pol I transcription is actively shut down and the nucleolus disappears. It is at this point that the specialized nature of active rRNA gene chromatin is most dramatically revealed by the appearance of NORs as secondary constrictions on metaphase chromosomes (McClintock 1934).

\section{Regulation by dosage compensation}

The level of rRNA gene transcription in eukaryotic cells is tightly regulated according to the protein synthesis requirements of the cell. Given the repetitive nature of these genes, one can imagine two strategies for regulating rRNA synthesis. The transcriptional level of active genes could be modulated, or alternatively, the number of active genes could vary. Short-term regulation of transcription in response to shifts in nutritional status, growth factor signaling, or stress, in organisms ranging from yeast to mammals is clearly modulated by altering the transcription level of active repeats. The principal target for this regulation appears to be the Pol I transcrip- 
tion factor Rrn3 in yeast or TIF-IA in mammals (Grummt 2003; Claypool et al. 2004).

There also is strong evidence in support of a form of long-term regulation in which the proportion of active genes is tightly controlled. The following are a few examples of this form of regulation, sometimes referred to as dosage compensation. Chicken cells that are di-, tri-, or tetrasomic for NOR-bearing chromosomes have the same level of rRNA synthesis despite varying greatly in rRNA gene content (Muscarella et al. 1985). Inbred lines of Maize can vary 10 -fold in rRNA gene content with no apparent differences in growth (Rivin et al. 1986). As we will see below, nucleolar dominance is another form of dosage compensation. Evidence that two classes of rRNA genes exist even in rapidly growing cells also comes from in vivo psoralen cross-linking experiments in yeast and mammals (Conconi et al. 1989; Dammann et al. 1995). One class of genes lacks regularly spaced nucleosomes over the transcribed region, and is accessible to the DNA cross-linking agent psoralen. The other class is packaged as nucleosomes, and is not accessible to psoralen. Here, I will discuss recent work regarding the differences in chromatin structure between active and silent rRNA genes. Special attention will be paid to the significant contributions that studies of nucleolar dominance in plant hybrids have made, including that of Earley et al. (2006). But first, some more background.

\section{NORs and secondary constrictions}

Metaphase NORs have the potential to provide a readout of the chromosomal distribution of active versus silent rRNA genes. Active NORs were initially identified as weakly stained chromatin regions on metaphase chromosomes around which nucleoli formed at the end of telophase (McClintock 1934). Such NORs are often referred to as secondary constrictions, the primary constrictions being centromeres. An axis of condensed chromatin is often found within the secondary constrictions on vertebrate chromosomes. This axis may perform a scaffolding role, and contains AT-rich DNA sequences as revealed by staining with dyes that bind preferentially to such DNA (Saitoh and Laemmli 1994). Staining of metaphase chromosomes with dyes that bind preferentially to GC-rich sequences (including rRNA genes) reveals the presence of less condensed sequences surrounding the axis of the secondary constriction (Saitoh and Laemmli 1994). These decondensed fibers have also been observed by electron microscopy, and the chromatin within them is calculated to be 10-fold less condensed than the remainder of the chromosome (Heliot et al. 1997; Suja et al. 1997). These fibers are comprised of rRNA genes that were transcriptionally active in the previous interphase, and that will be reactivated in the subsequent cell cycle. The most persuasive evidence that the presence of secondary constrictions correlates with transcriptional competence is that vertebrate Pol I transcription factors, including Upstream Binding Factor (UBF), remain associated throughout metaphase (Roussel et al. 1993, 1996).
Indeed, recent evidence suggests that extensive binding of UBF is sufficient to induce formation of a secondary constriction (Mais et al. 2005). The fact that a number of components of the Pol I transcription machinery contain acidic/argyophilic domains explained another longstanding observation, namely that metaphase NORs could be easily visualized by silver nitrate staining procedures (Goodpasture and Bloom 1975). Electron tomography performed on a silver-stained human metaphase NOR has led to a model in which the decondensed rRNA genes are organized as a single twisted loop that is anchored on the axis of the secondary constriction (Heliot et al. 1997).

Silent NORs do not appear as secondary constrictions, do not have associated Pol I transcription factors, and do not stain with silver. Even rapidly growing and dividing transformed human cell lines have silent NORs that are apparently fully condensed during mitosis (Roussel et al. 1996). Thus, in organisms that contain multiple NORs it appears that mechanisms exist to silence an entire NOR. The phenomenon of nucleolar dominance proves this point (see below). Variation in rRNA gene condensation state can also be observed within individual metaphase NORs. This is especially clear in the large NORs of rye, where sequential silver staining and fluorescence in situ hybridization (FISH) with an rRNA gene probe reveal condensed rRNA genes located adjacent to a secondary constriction containing decondensed rRNA genes that are positive in silver staining (Caperta et al. 2002). In the smaller NORs of vertebrates the situation is less clear. Nonetheless, evidence has been presented for the existence of condensed rRNA genes immediately adjacent to the axis of the secondary constriction (Suja et al. 1997).

\section{Nucleolar dominance}

Nucleolar dominance is a phenomenon observed in cells of interspecific hybrids in which NORs derived from one parental species are dominant over the other. Nucleolar dominance provides a powerful model for analyzing dosage compensation by biochemical approaches, since the rRNA genes and transcripts from each species can be distinguished. In the same year that McClintock (1934) determined that secondary constrictions corresponded to active NORs, Navashin (1934) observed in hybrids of the plant genus Crepis what is now described as nucleolar dominance. Each pure Crepis species has a single chromosome with a NOR that forms a secondary constriction. In hybrids, however, the NORs derived from only one species form secondary constrictions. Furthermore, the NORs derived from a number of species could be organized into a dominance hierarchy. These observations established nucleolar dominance as an epigenetic phenomenon. In subsequent years, nucleolar dominance has been described in many plant hybrids, including Brassica (Chen and Pikaard 1997b) and Arabidopsis (Chen et al. 1998). A similar phenomenon occurs in both Drosophila hybrids (Durica and Krider 1977) and Xenopus hybrids, where it was confirmed that differential 
rRNA gene transcription is at the basis of nucleolar dominance (Honjo and Reeder 1973). Finally, a form of nucleolar dominance is observed in human-mouse somatic cell hybrids (Croce et al. 1977).

The Pol I transcription machinery can diverge rapidly between species. The dedicated nature of the Pol I transcription machinery means that it evolves in concert with rRNA genes independent of other gene classes. Efficient transcription of rRNA genes by Pol I in vertebrates requires the formation of a preinitiation complex (PIC) on the promoter that includes UBF and a complex termed selectivity factor (SL1) in humans or TIF-1B in the mouse (Grummt 2003; Russell and Zomerdijk 2005). SL1/TIF-1B interacts with promoter DNA in a highly sequence-specific manner. PICs recruit an initiation competent subfraction of Pol I, defined by the presence of TIF-IA/Rrn3, the principal target of short-term regulation. Differences between mouse and human SL1/TIF$1 \mathrm{~B}$ are responsible for nucleolar dominance observed in mouse/human hybrid cell lines (Heix and Grummt 1995). Nucleolar dominance observed in Xenopus hybrids cannot be explained by this form of species specificity. In this case, it appears that differences in the number of enhancer elements found in the IGS of each species are responsible. The dominant NOR is that containing the greater number of enhancer repeats. This is the so-called "enhancer imbalance" hypothesis (Reeder 1985).

Neither species specificity of transcription factors nor "enhancer imbalance" can explain nucleolar dominance in plants. The basis of NOR selection in plant hybrids is currently unclear (Pikaard 2000). Despite this, the study of plant hybrids has provided a wealth of information about how nucleolar dominance is maintained epigenetically, and it is reasonable to assume that it will provide insights into how dominance is established. Furthermore, nucleolar dominance provides an excellent model system for understanding dosage compensation within pure species.

\section{Maintenance of silence, lessons from plants}

DNA methylation and histone modification play a central role in the maintenance of nucleolar dominance in allotetraploids of the genus Brassica. In plants and other higher eukaryotic organisms that methylate their genome, DNA methylation occurs on cytosines present in CpG or CpNpG motifs. The current wisdom is that DNA hypomethylation and histone hyperacetylation correlate with transcriptional activity, and that DNA hypermethylation and histone hypoacetylation correlate with transcriptional silencing. A role for DNA methylation in nucleolar dominance was dramatically revealed when seeds of Brassica allotetraploids were germinated in media containing 5-aza-2'-deoxycytodine (aza-dC), a nucleotide analog that inhibits cytosine methylation (Chen and Pikaard 1997a). This treatment resulted in transcriptional derepression of the silent rRNA genes to levels comparable with the dominant genes. Remark- ably, germination of seedlings in media containing histone deacetylase (HDAC) inhibitors sodium butyrate or trichostatin A (TSA) had the same effect.

Arabidopsis suecica is a naturally occurring hybrid of Arabidopsis thaliana and Arabidopsis arenosa (previously called Cardamonopsis arenosa). In these plants the A. thaliana rRNA genes are transcriptionally silent. As in Brassica, these silent genes can be reactivated by azadC (Chen et al. 1998). The following observations indicate that this dominance cannot be explained by enhancer imbalance or species specificity of transcription factors. In synthetic hybrids, silencing is more variable, and requires two generations to be fully established. Furthermore, changes in rRNA gene dosage could alter the direction of dominance. In a tetraploid that contained three sets of $A$. thaliana chromosomes and one of $A$. arenosa, the direction of dominance is reversed in favor of the A. thaliana genes (Chen et al. 1998).

The availability of the Arabidopsis genome sequence, the development of RNA interference (RNAi), and refinements to chromatin immunoprecipitation (ChIP) have now led to a fuller description of how nucleolar dominance is maintained (see Fig. 1). Bisulfite mapping reveals that in $A$. thaliana there are two classes of the rRNA gene promoter (Lawrence et al. 2004). The majority are extensively methylated, and a minority are under methylated overall but completely unmethylated in the core promoter element. The Pikaard Laboratory (Lawrence et al. 2004) has utilized a derivative of ChIP, termed ChIP-chop PCR, in which immunoprecipitated DNA fragments are digested with the enzyme McrBC prior to PCR. McrBC is an endonuclease that cleaves

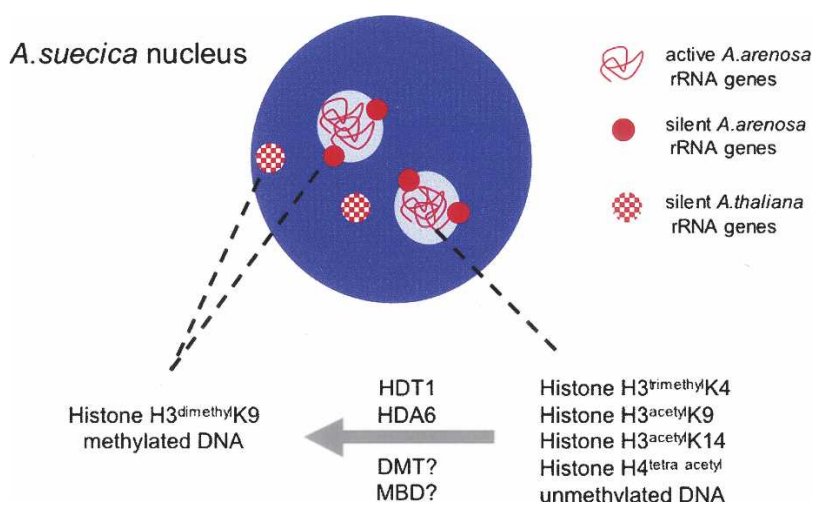

Figure 1. Distribution of silent and active rRNA genes in $A$. suecica nuclei. In the hybrid $A$. suecica, rRNA genes from the $A$. arenosa parental species are dominant over those from $A$. thaliana. Active genes within the $A$. arenosa NOR are decondensed, DNA-hypomethylated, and associated with the histone modifications shown on the right. Silent genes within the $A$. arenos $a$ NOR and the entire underdominant/silent $A$. thaliana NOR are condensed, DNA-hypermethylated, and associated with the histone modifications shown on the left. The nucleus is depicted in dark blue, with two nucleoli in pale blue. Histone deacetylase HDA6, a presumptive deacetylase HDT1, unidentified DMTs and MBD proteins are required for maintaining silence. 
DNA only when it is methylated at two or more cytosines. ChIP-chop PCR has shown that the archetypal epigenetic mark of heterochromatin, dimethylation of histone $\mathrm{H} 3$ on Lys $9\left(\mathrm{H} 3^{\text {dimethyl }} \mathrm{K} 9\right)$, associates with methylated (presumed silent) rRNA gene promoters. Conversley, Pol I and histone H3 trimethylated on Lys 4 $\left(\mathrm{H} 3^{\text {trimethyl }} \mathrm{K} 4\right)$ associate with unmethylated active promoters, establishing this as a mark for active chromatin.

In $A$. suecica hybrids, the dominant $A$. arenosa-derived NORs comprise a mixture of genes bearing either methylated promoters with associated $\mathrm{H} 3{ }^{\text {dimethyl }} \mathrm{K} 9$ or unmethylated promoters with $\mathrm{H} 3^{\text {trimethyl }} \mathrm{K} 4$ (Lawrence et al. 2004). Promoters in the underdominant $A$. thaliana NORs are uniformly methylated, associated with $\mathrm{H} 3{ }^{\text {dimethyl }} \mathrm{K}$ 9, and appear as condensed heterochromatin in the nuclei of meristematic root-tip cells. Treatment of hybrids with either TSA or aza-dC results in loss of DNA methylation at the $A$. thaliana promoters, loss of $\mathrm{H} 3{ }^{\text {dimethyl }} \mathrm{K}$ 9, gain of $\mathrm{H} 3^{\text {trimethyl }} \mathrm{K} 4$, and transcriptional activation.

Having characterized the epigenetic marks for silent and active rRNA genes/NORs, the focus changes to identifying the enzymatic activities responsible. The Arabidopsis genome is predicted to encode 16 HDACs, 12 histone acetyl-transferases (HATs), 10 cytosine methyltransferases (DMTs), and 12 methylcytosine-binding domain (MBD) proteins. The HDACs can be further subdivided into three families: the plant-specific HDT family, the RPD3-like HDA family, and the SIR2-like family (Pandey et al. 2002). Maize HD2, the founding member of the HDT family, is an acidic nucleolar phosphoprotein that is tightly bound to chromatin (Lusser et al. 1997). Arabidopsis HDT1 is also nucleolar, and plays a role in rRNA gene silencing (Lawrence et al. 2004). Underdominant $A$. thaliana genes are derepressed in HDT1-RNAi lines of $A$. suecica. Derepression correlates with a switch in the epigenetic marks from those of a repressed state, cytosine methylation, and $\mathrm{H} 3{ }^{\text {dimethyl }} \mathrm{K}$ 9, to those of an active state, promoter hypomethylation, $\mathrm{H} 3^{\text {trimethyl }} \mathrm{K} 4$, and histone H3 Lys 9 acetylation ( $\mathrm{H} 3{ }^{\text {acetyl }} \mathrm{K}$ 9).

The RPD/HDA family are so called based on their homology with yeast RPD3 and mammalian HDAC1. HDA6, a member of this family, has been identified in a number of genetic screens based on the reactivation of a transgene reporter. Two mutant alleles of HDA6 identified in these screens, sil1 and axe1-5, cause decondensation of rRNA gene chromatin (Probst et al. 2004). FISH performed on $A$. thaliana nuclei with an rRNA gene specific probe detects the silent genes, preferentially due to the higher target concentration present in condensed chromatin. These foci of condensed chromatin are deficient in acetylated histone $\mathrm{H} 4$ and $\mathrm{H} 3{ }^{\text {trimethyl }} \mathrm{K} 4$. In plants with sil1 and axe1-5 alleles of HDA6, these foci now appear less condensed and are now enriched in both acetylated $\mathrm{H} 4$ and $\mathrm{H} 3{ }^{\text {trimethyl }} \mathrm{K} 4$. Moreover, this effect seems relatively specific for rRNA genes, as global changes in histone modification are only slightly affected.

The importance of HDA6 in rRNA gene silencing and nucleolar dominance was further confirmed in a system- atic RNAi-mediated knockdown of RPD3-like and SIR2related HDACs in the $A$. suecica hybrid. In this issue, Earley et al. (2006) report that knockdown of HDA6 results in transcriptional derepression of the $A$. thaliana genes concomitant with a transition from heterochromatin to euchromatin. A. thaliana genes lose promoter cytosine methylation and $\mathrm{H} 3^{\text {dimethyl }} \mathrm{K} 9$ and gain not only the euchromatic mark $\mathrm{H} 3{ }^{\text {trimethyl }} \mathrm{K} 4$ as previously described, but also the histone acetylations $\mathrm{H} 3^{\text {acetyl }} \mathrm{K} 9$, $\mathrm{H} 3{ }^{\text {acetyl }} \mathrm{K} 14$, and histone $\mathrm{H} 4$ acetylated on $\mathrm{K} 5$, K8, K12, and $\mathrm{K} 16$ ( $\left.\mathrm{H}^{\text {tetra-acetylation }}\right)$, thus further extending the model of active rRNA gene chromatin (Earley et al. 2006).

A biochemical characterization of HDA6 demonstrates that it is a TSA-sensitive HDAC that can remove all the above histone acetylations. This combined with the demonstration that HDA6 at least partially localizes to nucleoli argues that HDA6 acts directly on rRNA gene chromatin (Earley et al. 2006). The similarity of these results with those obtained by HDT1 RNAi are striking and suggest that these HDACs function in the same repression pathway, although no direct interaction between these proteins has been detected. Thus far, no HDAC activity (TSA-sensitive or otherwise) has been described for HDT1, suggesting it may perform another role in rRNA gene repression.

A consistent observation throughout this work on plant nucleolar dominance is that histone deacetylation and DNA methylation are upstream of each other in a self-reinforcing pathway. TSA-mediated inhibition of a HDAC, now identified as HDA6, results in loss of DNA methylation, and aza-dC-mediated inhibition of DNA methylation results in loss of $\mathrm{H} 3^{\text {dimethyl }} \mathrm{K} 9$. This suggests a physical linkage between these processes. This interdependence is not restricted to the rRNA gene promoter, but appears to extend across at least the IGS. In animal cells, the chromodomain protein HP1 binds specifically to $\mathrm{H} 3{ }^{\text {dimethyl }} \mathrm{K} 9$ and in turn this can recruit the histone methyltransferase SUV39H1 (Jenuwein and Allis 2001). One can easily envisage such a complex facilitating the spread of at least H3K9 methylation across chromatin. The Arabidopsis genome encodes only one HP-1 related protein, LHP1. It will be interesting to assess its role in nucleolar dominance, although it should be pointed out that LHP1 has a number of characteristics that distinguish it from HP1 (Mylne et al. 2006).

Critically, the question of how are NORs selected for silencing remains unanswered. A systematic analysis of DMTs and MBDs involved in rRNA gene repression/ nucleolar dominance will be important, as identification of complexes between HDACs and DMTs and/or MBDs may begin to answer this question. Identifying a MBD involved in nucleolar dominance may be of particular significance, since a factor with an MBD-like domain is critical for targeting rRNA gene silencing in mammals.

\section{Establisment of silence, lessons from mammals}

In mammalian cells, silent rRNA gene repeats are characterized by extensive DNA methylation (Stancheva et 
al. 1997; Santoro and Grummt 2001). More recently, however, it has been demonstrated that methylation at a single position $(-133)$ in the mouse rRNA gene promoter impairs binding of UBF, thereby preventing transcription initiation complex formation on a chromatin template (Santoro and Grummt 2001). In addition to cytosine methylation, repressed rRNA genes bear the heterochromatic epigenetic marks of histone $\mathrm{H} 3^{\text {dimethyl }} \mathrm{K} 9$ and the chromodomain protein HP1 (Santoro et al. 2002). The nucleolar remodeling complex NoRC, an ATP-dependent chromatin remodeling complex that interacts with DMTs and HDACs, provides a mechanism for the targeted placement of these epigenetic marks at the rRNA gene promoter in mouse cells (see Fig. 2).

In vertebrates, the rRNA gene promoter is immediately preceded by a transcriptional terminator sequence, $\mathrm{T}_{\mathrm{O}}$ in mammals, that is recognized by the DNA-binding factor Transcription Termination Factor I (TTF-I) (Evers et al. 1995). TTF-I is required for transcription termination at $\mathrm{T}_{0}$, and also at the principal site of transcription termination at the $3^{\prime}$ end of pre-rRNA. A role for TTF-I and $\mathrm{T}_{0}$ in chromatin remodeling at the rRNA gene promoter was uncovered when it was demonstrated that addition of TTF-I was required for in vitro transcription
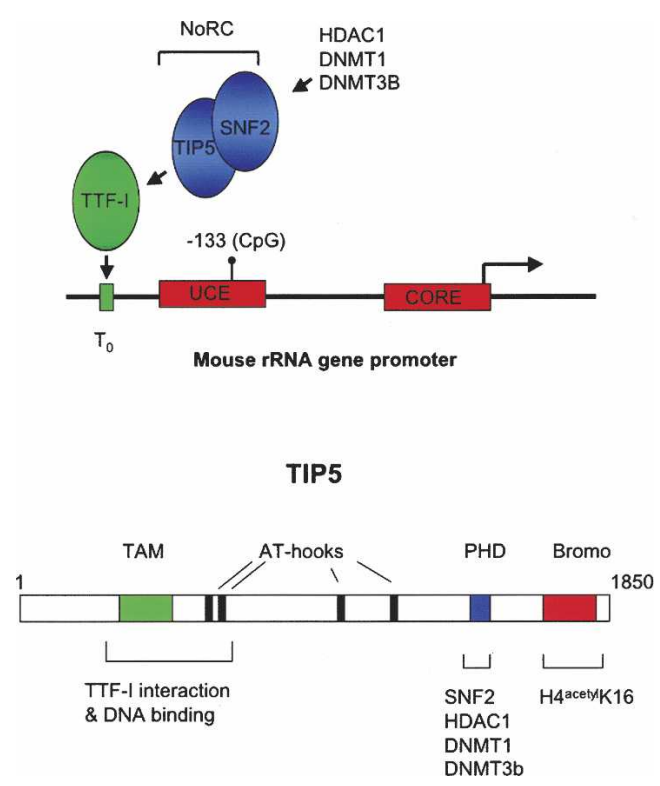

Figure 2. Recruitment of NoRC represses rRNA gene transcription in mammals. The mouse rRNA gene promoter contains two promoter elements: a CORE element centered over the transcription initiation site, and an upstream control element (UCE). Binding of the Pol I transcription factor UBF to the UCE is inhibited by CpG methylation at position -133. A promoter-proximal transcription terminator, $\mathrm{T}_{0}$, binds the factor TTF-I that in turn recruits the Nucleolar remodeling complex NoRC, resulting in transcriptional repression of the linked promoter. NoRC is comprised of TIP5 and the ATP-dependent remodeling factor SNF2. NoRC in turn recruits DNA methyltransferases DNMT1 and DNMT3b and the histone deacetylase HDAC1 that methylate promoter DNA and deacetylate promoter-bound histones (not shown), respectively. The domains of TIP5 that specify its various interactions are shown below. from a chromatinized mouse rRNA gene promoter (Langst et al. 1997). TTF-I recruits an ATP-dependent chromatin remodeling complex required for transcription initiation. A yeast two-hybrid-based search for proteins that interact with TTF-I identified TIP5 (TTF-Iinteracting protein 5), a nucleolar protein of $\sim 200 \mathrm{kDa}$ that shares a number of domains with the largest subunits of a number of other ATP-dependent chromatin remodeling complexes (Strohner et al. 2001). In common with these complexes, TIP5 is associated with the SNF2 ATPase. This complex of TIP5 and SNF2, termed NoRC, can induce nucleosomal movement along a DNA fragment in an ATP and histone H4 tail-dependent fashion. Surprisingly, rather than activating rRNA gene transcription, NoRC acts in repression. To date, an "activating" remodeling complex that associates with TTF-I remains undescribed.

NoRC components Tip5 and SNF2, together with HP1 and histone $\mathrm{H} 3{ }^{\text {methyl }} \mathrm{K} 9$, associate with methylated rRNA gene promoters (Santoro et al. 2002). Pol I and acetylated histone $\mathrm{H} 4$ associate with unmethylated/active promoters. NoRC is directly implicated in establishing these hetrochromatic marks, as overexpression of TIP5 results in de novo methylation and hypoacetylation of histone $\mathrm{H} 4$ on the promoter of a cotransfected reporter construct. Further evidence is provided by the observation that TIP5 can interact with DNA methyltransferases DNMT1 and DNMT3b, as well as the Sin3 corepressor complex that includes HDAC1 (Santoro et al. 2002). NoRC therefore has two functions: nucleosome remodeling, and acting as a scaffold for recruitment of DMTs and HDACs.

TIP5 contains a number of identifiable motifs that are important for its function. Most notable among these are the PHD (plant homeodomain) finger and an adjacent bromodomain that have been found in other transcriptional corepressors. Bromodomains are sequence elements, present in many chromatin-associated proteins and most HATs, that bind acetylated lysine residues within histones. The bromodomain of TIP5 interacts specifically with histone $\mathrm{H} 4^{\text {acetyl }} \mathrm{K} 16$ (Zhou and Grummt 2005). Binding to this acetylated residue is required for subsequent deacetylation of adjacent residues $\mathrm{K} 5, \mathrm{~K} 8$, and K12. Presumably, $4^{\text {acetyl }} \mathrm{K} 16$ is another epigenetic mark for silenced rRNA gene promoters. The TIP5 bromodomain cooperates with the adjacent PHD domain to recruit SNF2, HDAC1, DNMT1, and DNMT3b to rRNA gene promoters. Moreover, a fusion protein comprising these domains linked with the DNAbinding domain of TTF-1 is sufficient to induce silencing on a reporter plasmid. Other conserved domains of TIP5 include the TAM domain (TIP5/ARBD/MBD) and two adjacent AT hooks that are required for DNA binding and interaction with TTF-I (Strohner et al. 2001, 2004).

In plants, DNA methylation and histone deacetylation are interdependent. Importantly, this does not appear to be true of NoRC-dependent repression in mammalian cells. In this case, inhibition of histone deacetylation prevents DNA methylation while inhibition of DNA methylation does not affect histone modification (San- 
toro and Grummt 2005). Furthermore, epigenetic marks, particularly DNA methylation, that are targeted by the NoRC complex, appear to be restricted to the promoter. Yet in endogenous silenced gene repeats DNA methylation extends across the repeat (Santoro and Grummt 2001). Presumably, this requires additional activities that are independent of NoRC.

In most of the experiments described thus far, the effects of NoRC have been assayed on reporter constructs. This was for experimental reasons, so that the application of silencing could be tested on a fully active template. However, it is clear that TIP5 silences endogenous rRNA genes/NORs. Moderate overexpression of TIP5 in mouse cells silences rRNA gene expression, reduces the size and number of nucleoli, impairs cell proliferation, and shifts the replication timing of rRNA genes (Li et al. 2005). Replication of rRNA genes is biphasic. Active genes replicate early in $\mathrm{S}$ phase, and silent, NoRC-associated genes replicate late. Overexpression of TIP5 leads to a decrease in early replicating genes with a concomitant increase in the late replicating fraction. Replication timing, therefore, may be an important factor in maintaining the epigenetic marks of silence.

A number of issues remain unresolved regarding NoRC-dependent silencing of rRNA gene repeats. How does silencing, established at the promoter, spread across the repeat? An additional issue concerns the regulation of NoRC. The finding that TIP5 overexpression has such profound effects on rRNA gene transcription and cell growth implies that endogenous NoRC must be tightly regulated. It would be interesting to determine the nature of this regulation and contrast it with the shortterm regulation that targets the Pol I transcription factor TIF-IA.

\section{At what level does repression/dosage compensation occur?}

From studies of nucleolar dominance in plants and in animals it is abundantly clear that an entire NOR can be repressed as a unit. In mammals, however, NoRC-mediated repression clearly operates at the level of the promoter within each individual rRNA gene repeat. It is difficult to reconcile these two facts. For example, it is hard to envisage NoRC targeting every individual promoter within an individual NOR while leaving all the promoters within other NORs unaffected. Examination of metaphase NORs (by silver staining and/or staining with transcription factor antibodies) in response to overexpression or RNAi-mediated knockdown of NoRC may be very informative. If the number of active NORs alters, this would imply that NoRC can indeed regulate an entire NOR. Alternatively, if the staining intensity of a fixed number of NORs changes in response to altered NoRC activity, this would suggest that NoRC acted on individual, or at most, clusters of rRNA gene repeats.

Both NOR-based and promoter-based repression can be accommodated, however, if we propose that two classes of NORs exist: NORs in which the embedded rRNA genes are uniformly silent in a NoRC-independent manner, and a second class of NOR comprising a mosaic of active and silenced repeats. In this second class of NOR, the balance between active and silent genes is set by the level of NoRC activity in mammals or a related activity in plants. The evidence for mosaic NORs in plants is unequivocal. In interphase plant cells the usual pattern of an active NOR, visiualized by FISH, is diffuse intranucleolar labeling (active genes) emanating from perinucleolar heterochromatinized sites (silenced genes). This mosaicism can also be observed on metaphase chromosomes, and is especially clear on the large NORs of rye (Caperta et al. 2002). A number of vertebrates have only one NOR-bearing chromosome, and during interphase, diploid cells display two nucleoli (Robert-Fortel et al. 1993). In such cells, the NORs must be a mosaic of active and inactive genes, unless, of course, all the rRNA gene repeats are active. As the number of NOR-bearing chromosomes in an organism increases, the greater the possibility of achieving an appropriate balance of active versus inactive genes by a mechanism that silences entire NORs. In this case, NoRC may represent a mechanism of fine-tuning. At the moment, we do not know what the mechanism for silencing an entire NOR is, but nucleolar dominance should remain a fertile area of research.

\section{Acknowledgments}

I thank José-Luis Prieto and Jane Wright for comments on the manuscript. Work in my laboratory is supported by MRC UK.

\section{References}

Caperta, A.D., Neves, N., Morais-Cecilio, L., Malho, R., and Viegas, W. 2002. Genome restructuring in rye affects the expression, organization and disposition of homologous rDNA loci. J. Cell Sci. 115: 2839-2846.

Chen, Z.J. and Pikaard, C.S. 1997a. Epigenetic silencing of RNA polymerase I transcription: A role for DNA methylation and histone modification in nucleolar dominance. Genes \& Dev. 11: $2124-2136$.

. 1997b. Transcriptional analysis of nucleolar dominance in polyploid plants: Biased expression/silencing of progenitor rRNA genes is developmentally regulated in Brassica. Proc. Natl. Acad. Sci. 94: 3442-3447.

Chen, Z.J., Comai, L., and Pikaard, C.S. 1998. Gene dosage and stochastic effects determine the severity and direction of uniparental ribosomal RNA gene silencing (nucleolar dominance) in Arabidopsis allopolyploids. Proc. Natl. Acad. Sci. 95: 14891-14896.

Claypool, J.A., French, S.L., Johzuka, K., Eliason, K., Vu, L., Dodd, J.A., Beyer, A.L., and Nomura, M. 2004. Tor pathway regulates Rrn3p-dependent recruitment of yeast RNA polymerase I to the promoter but does not participate in alteration of the number of active genes. Mol. Biol. Cell 15: $946-$ 956.

Conconi, A., Widmer, R.M., Koller, T., and Sogo, J.M. 1989. Two different chromatin structures coexist in ribosomal rna genes throughout the cell cycle. Cell 57: 753-761.

Croce, C.M., Talavera, A., Basilico, C., and Miller, O.J. 1977. Suppression of production of mouse $28 \mathrm{~S}$ ribosomal RNA in mouse-human hybrids segregating mouse chromosomes. 
Proc. Nat1. Acad. Sci. 74: 694-697.

Dammann, R., Lucchini, R., Koller, T., and Sogo, J.M. 1995. Transcription in the yeast rRNA gene locus: Distribution of the active gene copies and chromatin structure of their flanking regulatory sequences. Mol. Cell. Biol. 15: 5294-5303.

Durica, D.S. and Krider, H.M. 1977. Studies on the ribosomal RNA cistrons in interspecific Drosophila hybrids. I. Nucleolar dominance. Dev. Biol. 59: 62-74.

Earley, K., Lawrence, R.J., Pontes, O., Reuther, R., Enciso, A.J., Silva, M., Neves, N., Gross, M., Viegas, W., and Pikaard, C.S. 2006. Erasure of histone acetylation by Arabidopsis HDA6 mediates large-scale gene silencing in nucleolar dominance. Genes \& Dev. (this issue).

Evers, R., Smid, A., Rudloff, U., Lottspeich, F., and Grummt, I. 1995. Different domains of the murine RNA polymerase Ispecific termination factor mTTF-I serve distinct functions in transcription termination. EMBO J. 14: 1248-1256.

Flaus, A. and Owen-Hughes, T. 2001. Mechanisms for ATPdependent chromatin remodelling. Curr. Opin. Genet. Dev. 11: $148-154$.

Goodpasture, C. and Bloom, S.E. 1975. Visualization of nucleolar organizer regions im mammalian chromosomes using silver staining. Chromosoma 53: 37-50.

Granneman, S. and Baserga, S.J. 2004. Ribosome biogenesis: Of knobs and RNA processing. Exp. Cell Res. 296: 43-50.

Grummt, I. 1999. Regulation of mammalian ribosomal gene transcription by RNA polymerase I. Prog. Nucleic Acid Res. Mol. Biol. 62: 109-154.

- 2003. Life on a planet of its own: Regulation of RNA polymerase I transcription in the nucleolus. Genes \& Dev. 17: 1691-1702.

Heix, J. and Grummt, I. 1995. Species specificity of transcription by RNA polymerase I. Curr. Opin. Genet. Dev. 5: 652656.

Heliot, L., Kaplan, H., Lucas, L., Klein, C., Beorchia, A., DocoFenzy, M., Menager, M., Thiry, M., O'Donohue, M.F., and Ploton, D. 1997. Electron tomography of metaphase nucleolar organizer regions: Evidence for a twisted-loop organization. Mol. Biol. Cell 8: 2199-2216.

Honjo, T. and Reeder, R.H. 1973. Preferential transcription of Xenopus laevis ribosomal RNA in interspecies hybrids between Xenopus laevis and Xenopus mulleri. J. Mol. Biol. 80: 217-228.

Jenuwein, T. and Allis, C.D. 2001. Translating the histone code. Science 293: 1074-1080.

Langst, G., Blank, T.A., Becker, P.B., and Grummt, I. 1997. RNA polymerase I transcription on nucleosomal templates: The transcription termination factor TTF-I induces chromatin remodeling and relieves transcriptional repression. EMBO $J$. 16: $760-768$.

Lawrence, R.J., Earley, K., Pontes, O., Silva, M., Chen, Z.J., Neves, N., Viegas, W., and Pikaard, C.S. 2004. A concerted DNA methylation/histone methylation switch regulates rRNA gene dosage control and nucleolar dominance. Mol. Cell 13: 599-609.

Li, J., Santoro, R., Koberna, K., and Grummt, I. 2005. The chromatin remodeling complex NoRC controls replication timing of rRNA genes. EMBO I. 24: 120-127.

Lusser, A., Brosch, G., Loidl, A., Haas, H., and Loidl, P. 1997. Identification of maize histone deacetylase HD2 as an acidic nucleolar phosphoprotein. Science 277: 88-91.

Mais, C., Wright, J.E., Prieto, J.L., Raggett, S.L., and McStay, B. 2005. UBF-binding site arrays form pseudo-NORs and sequester the RNA polymerase I transcription machinery. Genes \& Dev. 19: 50-64.
McClintock, B. 1934. The relatioship of a particular chromosomal element to the development of the nucleoli in Zea Mays. Z. Zellforsch. Mikrosk. Anat. 21: 294-328.

Miller Jr., O.L. and Beatty, B.R. 1969. Visualization of nucleolar genes. Science 164: 955-957.

Muscarella, D.E., Vogt, V.M., and Bloom, S.E. 1985. The ribosomal RNA gene cluster in aneuploid chickens: Evidence for increased gene dosage and regulation of gene expression. $J$. Cell Biol. 101: 1749-1756.

Mylne, J.S., Barrett, L., Tessadori, F., Mesnage, S., Johnson, L., Bernatavichute, Y.V., Jacobsen, S.E., Fransz, P., and Dean, C. 2006. LHP1, the Arabidopsis homologue of HETEROCHROMATIN PROTEIN1, is required for epigenetic silencing of FLC. Proc. Natl. Acad. Sci. 103: 5012-5017.

Navashin, M. 1934. Chromosome alterations caused by hybridisation and their bearing upon certain general genetic problems. Cytologia (Tokyo) 5: 169-203.

Pandey, R., Muller, A., Napoli, C.A., Selinger, D.A., Pikaard, C.S., Richards, E.J., Bender, J., Mount, D.W., and Jorgensen, R.A. 2002. Analysis of histone acetyltransferase and histone deacetylase families of Arabidopsis thaliana suggests functional diversification of chromatin modification among multicellular eukaryotes. Nucleic Acids Res. 30: 5036-5055.

Pikaard, C.S. 2000. The epigenetics of nucleolar dominance. Trends Genet. 16: 495-500.

Probst, A.V., Fagard, M., Proux, F., Mourrain, P., Boutet, S., Earley, K., Lawrence, R.J., Pikaard, C.S., Murfett, J., Furner, I., et al. 2004. Arabidopsis histone deacetylase HDA6 is required for maintenance of transcriptional gene silencing and determines nuclear organization of rDNA repeats. Plant Cell 16: $1021-1034$.

Reeder, R.H. 1985. Mechanisms of nucleolar dominance in animals and plants. J Cell Biol. 101: 2013-2016.

Rivin, C.J., Cullis, C.A., and Walbot, V. 1986. Evaluating quantitative variation in the genome of Zea mays. Genetics 113: 1009-1019.

Robert-Fortel, I., Junera, H.R., Geraud, G., and Hernandez-Verdun, D. 1993. Three-dimensional organization of the ribosomal genes and Ag-NOR proteins during interphase and mitosis in PtK1 cells studied by confocal microscopy. Chromosoma 102: 146-157.

Roussel, P., Andre, C., Masson, C., Geraud, G., and Hernandez, V.D. 1993. Localization of the RNA polymerase I transcription factor hUBF during the cell cycle. J. Cell Sci. 104: 327337.

Roussel, P., Andre, C., Comai, L., and Hernandez-Verdun, D. 1996. The rDNA transcription machinery is assembled during mitosis in active NORs and absent in inactive NORs. $J$. Cell Biol. 133: 235-246.

Russell, J. and Zomerdijk, J.C. 2005. RNA-polymerase-I-directed rDNA transcription, life and works. Trends Biochem. Sci. 30: 87-96.

Saitoh, Y. and Laemmli, U.K. 1994. Metaphase chromosome structure: Bands arise from a differential folding path of the highly AT-rich scaffold. Cell 76: 609-622.

Santoro, R. and Grummt, I. 2001. Molecular mechanisms mediating methylation-dependent silencing of ribosomal gene transcription. Mol. Cell 8: 719-725.

- 2005. Epigenetic mechanism of rRNA gene silencing: Temporal order of NoRC-mediated histone modification, chromatin remodeling, and DNA methylation. Mol. Cell. Biol. 25: 2539-2546.

Santoro, R., Li, J., and Grummt, I. 2002. The nucleolar remodeling complex NoRC mediates heterochromatin formation and silencing of ribosomal gene transcription. Nat. Genet. 32: 393-396. 
McStay

Stancheva, I., Lucchini, R., Koller, T., and Sogo, J.M. 1997. Chromatin structure and methylation of rat rRNA genes studied by formaldehyde fixation and psoralen cross-linking. Nucleic Acids Res. 25: 1727-1735.

Strohner, R., Nemeth, A., Jansa, P., Hofmann-Rohrer, U., Santoro, R., Langst, G., and Grummt, I. 2001. NoRC-A novel member of mammalian ISWI-containing chromatin remodeling machines. EMBO J. 20: 4892-4900.

Strohner, R., Nemeth, A., Nightingale, K.P., Grummt, I., Becker, P.B., and Langst, G. 2004. Recruitment of the nucleolar remodeling complex NoRC establishes ribosomal DNA silencing in chromatin. Mol. Cell. Biol. 24: 1791-1798.

Suja, J.A., Gebrane-Younes, J., Geraud, G., and Hernandez-Verdun, D. 1997. Relative distribution of rDNA and proteins of the RNA polymerase I transcription machinery at chromosomal NORs. Chromosoma 105: 459-469.

Zhou, Y. and Grummt, I. 2005. The PHD finger/bromodomain of NoRC interacts with acetylated histone H4K16 and is sufficient for rDNA silencing. Curr. Biol. 15: 1434-1438. 


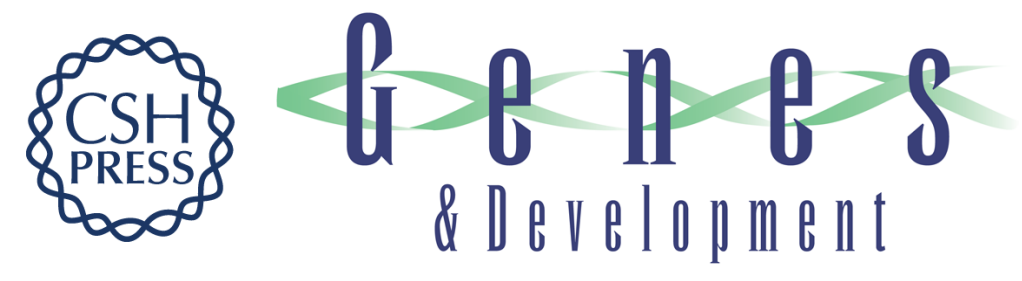

\section{Nucleolar dominance: a model for rRNA gene silencing}

Brian McStay

Genes Dev. 2006, 20:

Access the most recent version at doi:10.1101/gad.1436906

\section{Related Content Erasure of histone acetylation by Arabidopsis HDA6 mediates large-scale gene silencing in nucleolar dominance \\ Keith Earley, Richard J. Lawrence, Olga Pontes, et al. \\ Genes Dev. May , 2006 20: 1283-1293}

References This article cites 47 articles, 24 of which can be accessed free at:

http://genesdev.cshlp.org/content/20/10/1207.full.html\#ref-list-1

Articles cited in:

http://genesdev.cshlp.org/content/20/10/1207.full.html\#related-urls

\section{License}

Email Alerting

Service

Receive free email alerts when new articles cite this article - sign up in the box at the top right corner of the article or click here.

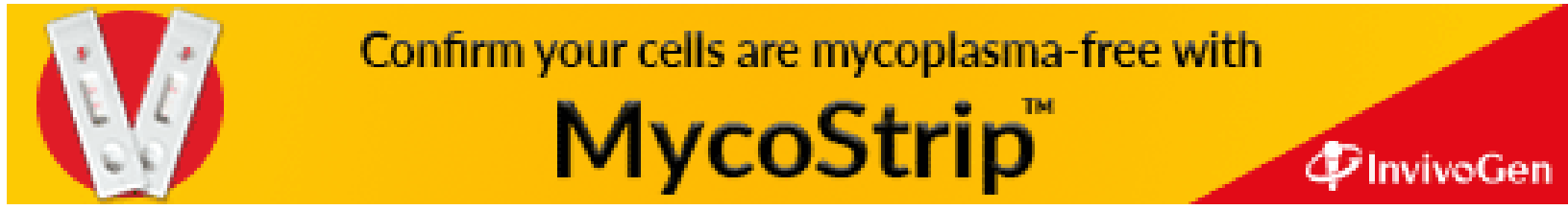

